\title{
Observation of Raman Optical Activity by Heterodyne-Detected Polarization-Resolved Coherent Anti-Stokes Raman Scattering
}

\author{
Kotaro Hiramatsu, ${ }^{1}$ Masanari Okuno, ${ }^{1}$ Hideaki Kano, ${ }^{2,1, *}$ Philippe Leproux, ${ }^{3}$ \\ Vincent Couderc, ${ }^{3}$ and Hiro-o Hamaguchi ${ }^{1,4, \dagger}$ \\ ${ }^{1}$ Department of Chemistry, School of Science, The University of Tokyo, 7-3-1 Hongo, Bunkyo-ku, Tokyo 113-0033, Japan \\ ${ }^{2}$ Institute of Applied Physics, University of Tsukuba, 1-1-1 Tennodai, Tsukuba, Ibaraki 305-8573, Japan \\ ${ }^{3}$ Institut de Recherche XLIM, UMR CNRS, No. 6172123 avenue Albert Thomas, 87060 Limoges cedex, France \\ ${ }^{4}$ Institute of Molecular Science and Department of Applied Chemistry, National Chiao Tung University,
}

1001 Ta Hsueh Road, Hsinchu 300, Taiwan

(Received 25 April 2012; published 24 August 2012)

\begin{abstract}
We report the first observation of Raman optical activity (ROA) by coherent anti-Stokes Raman scattering. Thanks to the more freedom of polarization configurations in coherent anti-Stokes Raman scattering than in spontaneous Raman spectroscopy, the contrast ratio of the chiral signal to the achiral background has been improved markedly. For (-)- $\beta$-pinene, it is 2 orders of magnitude better than that in the reported spontaneous ROA measurement. This is also the first measurement of ROA signal using a pulsed laser source.
\end{abstract}

Vibrational optical activity (VOA) such as vibrational circular dichroism (VCD) and Raman optical activity (ROA) has been able to provide a powerful method to determine the absolute configuration of chiral molecules and investigate the molecular conformation of biomolecules [1-5]. Time-resolved VOA, which combines VOA and the pump-probe technique, has a potential to trace the structural evolution of chiral molecules during various biochemical reactions. The pulsed measurements of VCD and vibrational optical rotatory dispersion (VORD) have already been reported using a femtosecond laser source [6]. On the other hand, ROA measurements with pulsed lasers have not been performed so far. In typical ROA measurements, small (typically $10^{-3}$ of the Raman intensity) intensity differences between the right- and left-circularly polarized incident or/and scattered Raman radiation are measured. This small ROA signal to achiral background ratio makes the time-resolved ROA measurements difficult because the signal is easily overwhelmed by background fluctuation.

One possible solution to this problem is to employ nonlinear Raman processes such as coherent anti-Stokes Raman scattering (CARS). ROA measurements with CARS have been proposed by Bjarnason et al. and Oudar et al. theoretically $[7,8]$. The CARS-ROA approach detects CARS radiation originating from the third-order optical susceptibility components that are negligible in electric dipole approximation but significant in the framework including the electric dipole-electric quadrupole or electric dipole-magnetic dipole contributions. In the CARS-ROA scheme, the achiral background can be suppressed by polarization-selective CARS measurements, because the polarization of chirality-induced CARS signals is orthogonal to that of the achiral background. Moreover,
CARS-ROA should be more advantageous than spontaneous ROA in the aspect of accessibility to fluorescent samples. However, CARS-ROA signals are estimated to be about 6 orders of magnitude smaller than the typical achiral CARS signals [8]. Therefore, a CARS spectrometer with a high sensitivity and polarization selectivity is necessary for the CARS-ROA measurement. Recently, a multiplex CARS technique evolved rapidly with the use of a broadband white-light laser pulse generated from a photonic crystal fiber (PCF) [9-11]. Multiplex CARS spectra with a high signal to noise ratio can now be obtained without wavelength scanning [12]. In the present Letter, we report the observation of the CARS-ROA signal with heterodyne-detected polarization-resolved multiplex CARS spectroscopy. To the best of our knowledge, this is the first report of CARS-ROA, as well as the first ROA observation with a pulsed laser source.

The third-order optical susceptibility of the CARS process is given by the sum of the nonresonant background and the vibrationally resonant terms,

$$
\chi_{i j k l}\left(2 \omega_{1}-\omega_{2} ; \omega_{1}, \omega_{1},-\omega_{2}\right)=\chi_{i j k l}^{\mathrm{NR}}+\chi_{i j k l}^{R} .
$$

The vibrationally resonant part is written as

$$
\chi_{i j k l}^{R}=\frac{N X_{i j k l}}{2 \hbar\left(\Omega-\omega_{1}+\omega_{2}-i \Gamma\right)},
$$

where $N$ is the number of molecules per unit volume, $\Omega$ is the frequency of a Raman active vibrational mode, $\omega_{1}$ and $\omega_{2}$ are the frequencies of the pump and Stokes incident radiation, respectively, and $\Gamma$ is a phenomenological damping constant. In the electric dipole approximation, $X_{1111}$ and $X_{2111}$ can be written as $X_{1111}=2 a^{2}+8 \gamma^{2} / 45$ and $X_{2111}=0$, where $a$ and $\gamma$ are the isotropic and anisotropic rotational invariants of the polarizability tensor, 
respectively. No information about molecular chirality is contained in these invariants. Beyond the electric dipole approximation, however, the electric dipole-electric quadrupole and electric dipole-magnetic dipole interactions give nonzero values of the $X_{2111}$ component for the optical activity tensor invariants $[7,8]$ :

$$
\begin{gathered}
X_{1111}=2 a^{2}+\frac{8 \gamma^{2}}{45}, \\
X_{2111}=-4 i n\left(a G^{\prime}+\frac{\gamma_{G}^{2}}{45}\right)+\frac{2 i n \gamma_{A}^{2}}{135 c}\left(1-\frac{\omega_{1}-\omega_{2}}{2 \omega_{1}}\right),
\end{gathered}
$$

where $G^{\prime}$ is the isotropic invariant of the electric dipolemagnetic dipole optical activity tensor products, $\gamma_{G}$ and $\gamma_{A}$ are the anisotropic invariants of the electric dipolemagnetic dipole and electric dipole-electric quadrupole optical activity tensor products, respectively [1].

In spontaneous ROA experiments, the dimensionless circular intensity difference (CID) is measured. CID is defined as $\Delta=\left(I_{R}-I_{L}\right) /\left(I_{R}+I_{L}\right)$. Here $I_{R}$ and $I_{L}$ are the Raman intensities obtained with the right- and left-circularly polarized incident light. The CID for forward $\left(0^{\circ}\right)$ scattering is written in terms of the tensor invariants $[1,13]$ :

$$
\Delta\left(0^{\circ}\right)=\frac{4 n\left(45 a G^{\prime}+\gamma_{G}^{2}-\gamma_{A}^{2} / 6 c\right)}{45 a^{2}+7 \gamma^{2}} .
$$

By comparing Eqs. (4) and (5), $X_{2111}$ is approximated by the product of $\Delta\left(0^{\circ}\right)$ and $X_{1111}$

$$
X_{2111} \simeq-\frac{i}{2} \Delta\left(0^{\circ}\right) X_{1111} \text {. }
$$

Thus, the third-order susceptibilities are expressed as

$$
\begin{aligned}
& \chi_{1111}=\chi_{1111}^{\mathrm{NR}}+\sum_{i=1}^{n} \frac{A_{i}}{\Omega_{i}-\omega_{1}+\omega_{2}-i \Gamma_{i}}, \\
& \chi_{2111} \simeq \chi_{2111}^{\mathrm{NR}}+\sum_{i=1}^{n} \frac{-i \Delta_{i}\left(0^{\circ}\right) A_{i} / 2}{\Omega_{i}-\omega_{1}+\omega_{2}-i \Gamma_{i}} .
\end{aligned}
$$

If the polarizations of the two incident beams are parallel to each other, the observed CARS spectrum is formulated as follows:

$$
\begin{aligned}
& I\left(\omega_{1}-\omega_{2}, \theta\right) \\
& \propto\left|\cos \left(\theta-\theta_{0}\right) \chi_{2111}+\sin \left(\theta-\theta_{0}\right) \chi_{1111}\right|^{2} \\
& =\cos ^{2}\left(\theta-\theta_{0}\right)\left|\chi_{2111}\right|^{2}+\sin ^{2}\left(\theta-\theta_{0}\right)\left|\chi_{1111}\right|^{2} \\
& \quad+2 \cos \left(\theta-\theta_{0}\right) \sin \left(\theta-\theta_{0}\right) \operatorname{Re}\left(\chi_{2111}^{*} \chi_{1111}\right),
\end{aligned}
$$

where the definition of the angle difference $\theta-\theta_{0}$ is depicted in Fig. 1(a). In the present study, $\theta_{0}$ is set to be close to zero. In Eq. (10), the first and second terms correspond to the homodyne-detected signal of the chiral
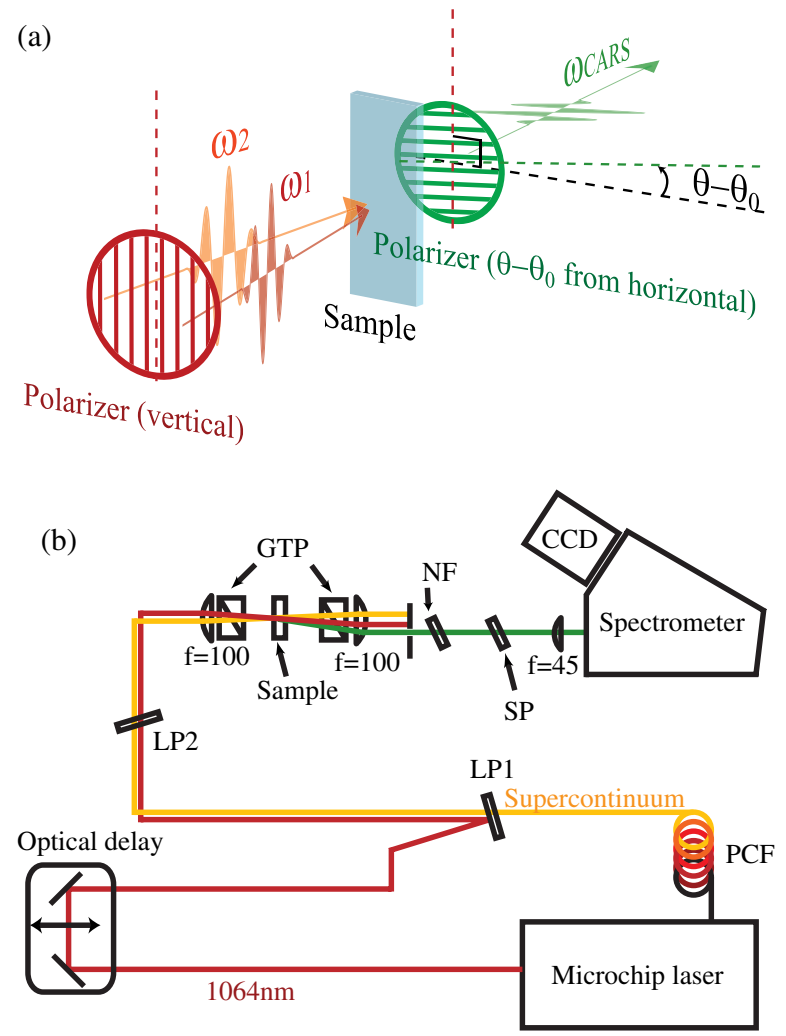

FIG. 1 (color online). (a) Schematics of the experimental configuration around the sample. $\theta$ represents the angle of the analyzer and $\theta_{0}$ is the angle at which the polarizers before and after the sample are perpendicular. (b) Schematic of our heterodyne-detected polarization-resolved CARS spectroscopic system: LP1, $1064 \mathrm{~nm}$ long-pass filter; LP2, $1050 \mathrm{~nm}$ long-pass filter; GTP, Gran-Taylor Prisms; NF, $1064 \mathrm{~nm}$ notch filter; SP, $1050 \mathrm{~nm}$ short-pass filter. The polarizations of the indent and CARS radiation are precisely (extinction ratio $\sim 1: 10^{-7}$ ) set by Gran-Taylor prisms.

and achiral components, respectively. The last cross term in Eq. (10) expresses the interference between the achiral and chiral signals. By using the achiral signal as a local oscillator (LO), the detection sensitivity to the chiral signal can be enhanced through the scheme of heterodyne detection. Heterodyne detection enables us to retrieve the phase of the chiral signal, which corresponds to the signs of $\Delta_{i}$ in Eq. (8). We can simply extract the cross term in Eq. (10) as a difference spectrum between $\theta=\theta_{0}+\theta_{1}$ and $\theta=\theta_{0}-\theta_{1}$ as

$$
\begin{aligned}
I\left(\omega_{1}\right. & \left.-\omega_{2}, \theta_{0}+\theta_{1}\right)-I\left(\omega_{1}-\omega_{2}, \theta_{0}-\theta_{1}\right) \\
& \propto 2 \sin \theta_{1} \cos \theta_{1} \operatorname{Re}\left(\chi_{2111}^{*} \chi_{1111}\right) \\
& =\sum_{i=1}^{n} \frac{2 \chi_{1111}^{\mathrm{NR}} \Delta_{i} A_{i} \Gamma_{i} \sin \theta_{1}}{\left(\Omega_{i}-\omega_{1}+\omega_{2}\right)^{2}+\Gamma_{i}^{2}} .
\end{aligned}
$$

In Eq. (12), $\chi_{2111}^{\mathrm{NR}}$ is assumed to be 0 for simplicity (see Supplemental Material [14]). On the other hand, the spontaneous ROA line shape is written as follows: 


$$
I_{R}(\omega)-I_{L}(\omega) \propto \sum_{i=0}^{n} \frac{\Delta_{i} A_{i} \Gamma_{i}}{\left(\Omega_{i}-\omega\right)^{2}+\Gamma_{i}^{2}} .
$$

Equations (12) and (13) suggest that the spontaneous ROA spectrum in the forward scattering and the difference CARS spectrum between $\theta=\theta_{0} \pm \theta_{1}$ provide the same spectral profile.

The schematic diagram of our heterodyne-detected polarization-resolved multiplex CARS system is shown in Fig. 1(b). The laser source is a passively Q-switched subnanosecond $1064 \mathrm{~nm}$ microchip laser with a repetition rate of $33 \mathrm{kHz}$. The output of the laser is divided into two. One is used as the narrow-band pump pulses of the CARS process. The other is introduced into a 6-m long air-silica PCF to generate a supercontinuum (white-light laser) with $>100 \mu \mathrm{W} / \mathrm{nm}$ spectral power density from 1.05 to $1.6 \mu \mathrm{m}$ and is used as the broadband Stokes pulses of the CARS process. After eliminating the anti-Stokes spectral component of the pump and the Stokes pulses with several filters, the two pulses propagate collinearly and are superimposed at the sample with an achromatic lens (AC254-100-C, Thorlabs). The polarizations of the two incident beams are set parallel to each other by a GranTaylor prism just before the sample. The polarization of the generated CARS field is selected by an analyzer (GranTaylor prism) just after the sample. For the precise polarization selection of the CARS field, the analyzer is mounted on a motorized rotational stage (SGSP-60YAW0B, Sigma Koki). The angle of the analyzer can be controlled with a minimum increment of $0.0025^{\circ}$ and repeatability of $0.02^{\circ}$. After selecting the polarization of the generated CARS field, the incident beams are spectrally blocked by a notch filter and a short-pass filter. Finally, the CARS radiation is introduced into a polychromator (LS785, Princeton Instruments), to be detected by a CCD camera (PIXIS 100BR eXcelon, Princeton Instruments). In order to evaluate our CARS-ROA system, we performed proof-of-principle experiments on liquid (-)- $\beta$-pinene. Liquid (-)- $\beta$-pinene was purchased from Aldrich and used as received. The sample was set in a quartz cell with a path length of $1.0 \mathrm{~mm}$. The pulse energies of the pump and Stokes radiation were $6 \mu \mathrm{J}$ and $2 \mu \mathrm{J}$, respectively.

Figure 2 shows a series of polarization-resolved CARS spectra of liquid (-)- $\beta$-pinene at the analyzer angle $\theta=$ $10.0^{\circ}$ and at $\theta=-0.075^{\circ}$ to $0.075^{\circ}$ with $0.025^{\circ}$ increments. The collection time for the CARS spectrum with $\theta=10.0^{\circ}$ is $100 \mathrm{~s}$ (100 times accumulation of one second exposure) and $30 \mathrm{~min}$ for the other spectra (from $\theta=-0.075^{\circ}$ to $0.075^{\circ}$ ). The spontaneous anti-Stokes Raman-scattering signal generated by the pump pulse was subtracted from all the spectra. After this subtraction, all the spectra were divided by the nonresonant background spectrum obtained from water to compensate the spectral profile of the Stokes pulse.

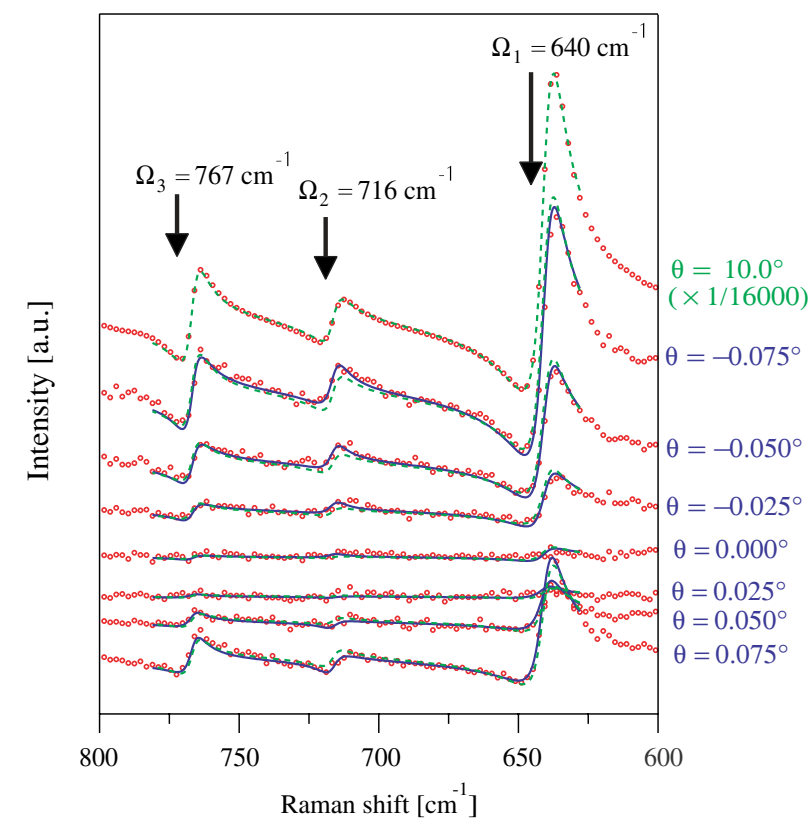

FIG. 2 (color online). Circles: A set of observed polarizationresolved CARS spectra of the liquid (-)- $\beta$-pinene [the collection time; $100 \mathrm{~s}$ (100 times accumulation of one second exposure) for the spectrum at $\theta=10.0^{\circ}, 30 \mathrm{~min}$ for the other spectra, the incident power; $\sim 200 \mathrm{~mW}$ for $\omega_{1}$ and $\sim 70 \mathrm{~mW}$ for $\left.\omega_{2}\right]$. The spectra are normalized by the collection time. The spectrum at $\theta=10.0^{\circ}$ is minified by $1 / 16000$. Dashed lines: Curves fitted by using Eq. (14) with $\chi_{2111}$ and $C_{\text {leak }}$ are fixed to be 0 . From the spectrum at $\theta=10.0^{\circ}$, the parameters were obtained as $\chi_{1111}^{\mathrm{NR}}=3.2 \times 10^{-14}[\mathrm{esu}], \omega_{i}=640.39 \pm 0.027,716.28 \pm 0.16$, $766.74 \pm 0.80\left[\mathrm{~cm}^{-1}\right], \quad A_{i}=(17.4 \pm 0.1) \times 10^{-14}, \quad(2.2 \pm 0.1) \times$ $10^{-14},(4.2 \pm 0.1) \times 10^{-14}[\mathrm{esu}], \Gamma_{i}=4.94 \pm 0.05,3.76 \pm 0.25$, $3.49 \pm 0.13\left[\mathrm{~cm}^{-1}\right](i=1,2,3$, respectively) Solid lines: Curves fitted by using Eq. (14) with free $\chi_{2111}$ and $C_{\text {leak }}$. From the spectra at $\theta=-0.075^{\circ},-0.050,{ }^{\circ} \ldots, 0.075^{\circ}$, the parameters were obtained as $\chi_{2111}^{\mathrm{NR}}=0 \pm 3 \times 10^{-19} \times 10^{-19}$ [esu], $C_{\text {main }}=(6.35 \pm 0.03) \times 10^{2}, \quad C_{\text {leak }}=(3.65 \pm 0.35) \times 10^{-6}$, $\theta_{0}=0.016^{\circ} \pm 0.0006^{\circ}, \quad \Delta_{1}=(0.38 \pm 0.03) \times 10^{-3}, \quad \Delta_{2}=$ $(-1.17 \pm 0.14) \times 10^{-3}, \Delta_{3}=(0.52 \pm 0.08) \times 10^{-3}$. The absolute magnitudes of the third-order optical susceptibilities are evaluated by using the value of the nonresonant third-order susceptibility of water as a standard [21].

Based on the previous report [15], the $716 \mathrm{~cm}^{-1}$ and $767 \mathrm{~cm}^{-1}$ Raman bands of (-)- $\beta$-pinene have large ROA in forward scattering. Therefore, we focus on the spectral region ranging from $600 \mathrm{~cm}^{-1}$ to $800 \mathrm{~cm}^{-1}$. We do not obtain the homodyne chiral component [the first term in Eq. (10)] around $\theta=0^{\circ}$, which indicates that the homodyne chiral signal is overwhelmed by the noise of the background signal. In order to extract the ROA signal, we analyze the spectra according to the following procedure. First, we determine the parameters $\chi_{1111}^{\mathrm{NR}}$ and $A_{i}, \Omega_{i}, \Gamma_{i}$ for the three peaks, where the indices $i=1,2,3$ denote the Raman modes at $640 \mathrm{~cm}^{-1}, 716 \mathrm{~cm}^{-1}$, and $767 \mathrm{~cm}^{-1}$, respectively, by fitting the CARS spectrum at $\theta=10.0^{\circ}$ with the second term in Eq. (10). For $\theta=10.0^{\circ}$, 
the chiral signal is negligible in comparison with the achiral signal (the second term $\gg$ the first and third terms). Next, using these parameters, all the seven spectra we observed from $\theta=-0.075^{\circ}$ to $\theta=0.075^{\circ}$ with $0.025^{\circ}$ increments are fitted simultaneously with the following equation:

$$
\begin{aligned}
I\left(\omega_{1}-\omega_{2}, \theta\right)= & C_{\text {main }}\left|\chi_{2111}+\sin \left(\theta-\theta_{0}\right) \chi_{1111}\right|^{2} \\
& +C_{\text {leak }}\left|\chi_{1111}\right|^{2},
\end{aligned}
$$

where $C_{\text {main }}$ and $C_{\text {leak }}$ represent the magnitudes of the spectral component properly oriented by the analyzer and the leaked signal of the large $\chi_{1111}$ signal due to the nonzero extinction ratio of the analyzer $\left(\sim 10^{-7}\right)$, respectively.

The least-squares-fitted results are shown as solid curves in Fig. 2. They agree with the observed spectra (shown as circles) very well. The optical activity parameters were determined as $\chi_{2111}^{\mathrm{NR}} / \chi_{1111}^{\mathrm{NR}}=0 \pm 1 \times 10^{-5}, \Delta_{1}=$ $(0.38 \pm 0.03) \times 10^{-3}, \Delta_{2}=(-1.17 \pm 0.14) \times 10^{-3}$, and $\Delta_{3}=(0.52 \pm 0.08) \times 10^{-3}$. It should be noted that $\Delta_{i}$ have significant nonzero values, and the signs and the order of these values are consistent with those obtained with the spontaneous ROA study [15]. The fitted results with $\Delta_{i}$ fixed to be zero are also shown as dashed curves in Fig. 2. In this case, the fitted results cannot reproduce the experimental results, in particular around $\Omega_{2}$. This fact suggests that the ROA components certainly contribute to the observed CARS spectra.

Based on these results, we can extract an ROA spectrum using Eq. (12). The sum and difference of CARS spectra between the $\theta=-0.075^{\circ}$ and $\theta=0.075^{\circ}$ spectra with appropriate coefficients are shown in Figs. 3(a) and 3(b). The coefficients are determined by using Eq. (10) so that the sum and difference of the spectra are proportional to $\left|\chi_{1111}\right|^{2}$ and $\operatorname{Re}\left(\chi_{2111}^{*} \chi_{1111}\right)$, respectively. Two ROA peaks at $716 \mathrm{~cm}^{-1}$ and $767 \mathrm{~cm}^{-1}$ are clearly observed in the spectrum obtained with this procedure. All the spectral

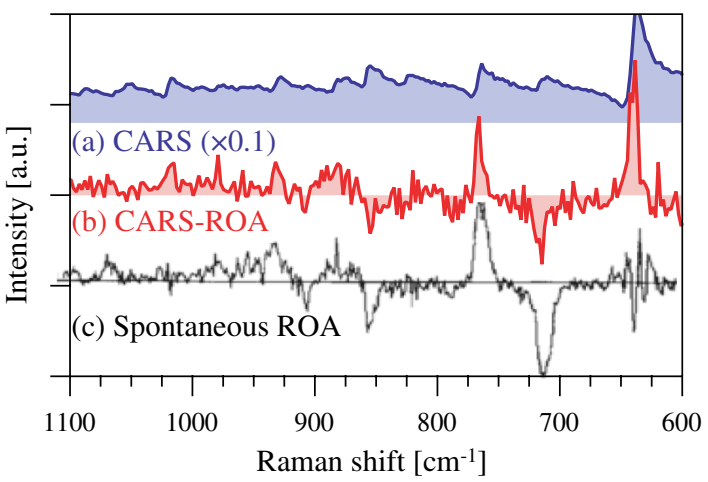

FIG. 3 (color online). (a): The sum of CARS spectra with weight coefficients: $\quad I_{\text {sum }}=I\left(0.075^{\circ}-\theta_{0}\right)+I\left(-0.075^{\circ}-\right.$ $\left.\theta_{0}\right) \sin \left(0.075^{\circ}-\theta_{0}\right) / \sin \left(-0.075^{\circ}-\theta_{0}\right)(\mathrm{b})$ : The difference of CARS spectra with weight coefficients: $I_{\text {dif }}=I\left(0.075^{\circ}-\right.$ $\left.\theta_{0}\right)-I\left(-0.075^{\circ}-\theta_{0}\right) \sin \left(0.075^{\circ}-\theta_{0}\right) / \sin \left(-0.075^{\circ}-\theta_{0}\right)$ (c): Spontaneous ROA spectrum reproduced from Ref. [15]. features in Fig. 3(b) except for the $640 \mathrm{~cm}^{-1}$ band quantitatively agree with those in the spontaneous ROA spectrum [Fig. 3(c)] obtained by Barron et al. [15]. The band at $640 \mathrm{~cm}^{-1}$ is strongly polarized and has been found to be unreliable in ROA studies $[13,16]$.

The contrast between the ROA signal and achiral background is determined by $|\Delta|=\left|\left(I_{R}-I_{L}\right) /\left(I_{R}+I_{L}\right)\right|$ in the spontaneous ROA measurement. Those of the $716 \mathrm{~cm}^{-1}$ and $767 \mathrm{~cm}^{-1}$ Raman bands of (-)- $\beta$-pinene are estimated to be around $1 \times 10^{-3}$. In our CARS-ROA measurement, the contrast is determined as $2 \operatorname{Re}\left(\chi_{2111}^{*} \chi_{1111}\right) /$ $\left(\sin \left(\theta-\theta_{0}\right)\left|\chi_{1111}\right|^{2}\right)$. Note that the contrast is the function of the parameter of $\theta$ that can be controlled experimentally. At $\theta=0.075^{\circ}$, the signal to background ratio experimentally determined by the ratios between the peak intensities of the CARS-ROA and CARS spectra are about $1 \times 10^{-1}$ for the same two peaks [Figs. 3(a) and 3(b)].

Besides CARS-ROA, several nonlinear spectroscopic techniques sensitive to molecular chirality have been proposed and developed. In particular, it is worthwhile to compare CARS-ROA with BioCARS, which is a chiralsensitive $\chi^{(4)}$ Raman spectroscopy proposed by Koroteev [17]. Like chiral $\chi^{(2)}$ spectroscopy [18,19], chiralityinduced signals can be obtained without achiral background in BioCARS because even-order nonlinear susceptibilities are zero in the achiral liquid. Up to the present, however, BioCARS has not been observed primarily due to its extremely small $\chi^{(4)}$ value (estimated to be $\left.10^{-22} \mathrm{esu}\right)$. To obtain BioCARS signal of the energy $6 \times 10^{-12} \mathrm{~J}$ in each pulse, incident pulses with $1 \mathrm{ps}$ pulse width and $50 \mu \mathrm{J}$ pulse energy focused into a spot with diameter $60 \mu \mathrm{m}$ are needed in electronically preresonant conditions [17]. In CARS-ROA, the magnitudes of the optically active third-order susceptibilities are estimated to be $\left|\chi_{2111}^{\mathrm{R}}\right|=\left|\Delta \chi_{1111}^{\mathrm{R}} / 2\right| \sim 10^{-17}$ esu in the off-resonance condition. To obtain the CARS-ROA pulse [the signal of the heterodyne term in Eq. (12)] with the same energy as discussed above $\left(6 \times 10^{-12} \mathrm{~J}\right.$ in each pulse), incident pulses with $1 \mathrm{ps}$ pulse width and only $2 \mu \mathrm{J}$ energy are necessary [20].

In summary, we have developed a new ROA technique based on coherent Raman spectroscopy, the heterodynedetected polarization-resolved CARS spectroscopy. This technique is applied to measure the Raman optical activity of liquid (-)- $\beta$-pinene successfully, and is the first reported on not only CARS-ROA but also on ROA using a pulsed laser. Thanks to the coherent nature of the CARS signal, the large achiral CARS electric field can be used as a local oscillator, and it could enable us to extract the amplitude and phase of a weak chiral signal. Our results show that the achiral background can be significantly reduced by adjusting polarizations, making the measurements robust over fluctuations of an optical system. This technique will be applied to time-resolved ROA measurements for unraveling the dynamics of transient chiral molecules and 
biomolecules in aqueous solutions to which infrared VCD is not applicable.

This work is supported by the SENTAN project (Program-S) of the Japan Science and Technology Agency (JST). H. Kano gratefully acknowledges financial support by Grand-Aid for Scientific Research on Priority "Molecular Science for Supra Functional Systems" [477] from MEXT, and the Global COE Program for "Chemistry Innovation." The authors thank the LEUKOS company for technical support with the dual-output supercontinuum light source. The authors gratefully acknowledge J. Ukon, HORIBA, Ltd. for assisting with a fruitful collaboration between Japanese and French labs.

*Corresponding author hkano@bk.tsukuba.ac.jp

†Corresponding author hhama@nctu.edu.tw

[1] L.D. Barron, Molecular Light Scattering and Optical Activity (Cambridge University Press, Cambridge, 2004).

[2] L. Nafie, Vibrational Optical Activity: Principles and Applications (Wiley, New York, 2011).

[3] J. Costante, L. Hecht, P. L. Polavarapu, A. Collet, and L. D. Barron, Angew. Chem., Int. Ed. Engl. 36, 885 (1997).

[4] R. Silva, J. Kubelka, P. Bour, S. M. Decatur, and T. A. Keiderling, Proc. Natl. Acad. Sci. U.S.A. 97, 8318 (2000).

[5] J. Haesler, I. Schindelholz, E. Riguet, C. G. Bochet, and W. Hug, Nature (London) 446, 526 (2007).

[6] H. Rhee, Y.-G. June, J.-S. Lee, K.-K. Lee, J.-H. Ha, Z. H. Kim, S.-J. Jeon, and M. Cho, Nature (London) 458, 310 (2009).
[7] J.O. Bjarnason, H.C. Andersen, and B.S. Hudson, J. Chem. Phys. 72, 4132 (1980).

[8] J. L. Oudar, C. Minot, and B. A. Garetz, J. Chem. Phys. 76, 2227 (1982).

[9] H. N. Paulsen, K. M. Hilligse, J. Thøgersen, S. R. Keiding, and J. J. Larsen, Opt. Lett. 28, 1123 (2003).

[10] H. Kano and H. Hamaguchi, Appl. Phys. Lett. 86, 121113 (2005).

[11] M. Okuno, H. Kano, P. Leproux, V. Couderc, J. P. R. Day, M. Bonn, and H.-o. Hamaguchi, Angew. Chem., Int. Ed. Engl. 49, 6773 (2010).

[12] P. Leproux, V. Couderc, A. de Angelis, M. Okuno, H. Kano, and H. Hamaguchi, J. Raman Spectrosc. 42, 1871 (2011).

[13] L. Hecht and L. D. Barron, Spectrochim. Acta, Part A 45, 671 (1989).

[14] See Supplemental Material at http://link.aps.org/ supplemental/10.1103/PhysRevLett.109.083901 for the case $\chi_{2111}^{\mathrm{NR}}$ that is not equal to zero.

[15] L. D. Barron, L. Hecht, A. R. Gargaro, and W. Hug, J. Raman Spectrosc. 21, 375 (1990).

[16] L. D. Barron and J. R. Escribano, Chem. Phys. Lett. 126, 461 (1986).

[17] N. Koroteev, Biospectroscopy 1, 341 (1995).

[18] N. Ji, V. Ostroverkhov, M. Belkin, Y.-J. Shiu, and Y.-R. Shen, J. Am. Chem. Soc. 128, 8845 (2006).

[19] M. A. Belkin, T. A. Kulakov, K. H. Ernst, L. Yan, and Y. R. Shen, Phys. Rev. Lett. 85, 4474 (2000).

[20] J.F. Reintjes, Nonlinear Optical Parametric Processes in Liquids and Gases (Academic Press, New York, 1984).

[21] R. L. Sutherland, Handbook of Nonlinear Optics (Marcel Dekker, New York, 1996). 